\title{
KLEIN SURFACES AND REAL ALGEBRAIC FUNCTION FIELDS ${ }^{1}$
}

\author{
BY NORMAN ALLING AND NEWCOMB GREENLEAF
}

Communicated by Serge Lang, February 21, 1969

Introduction. The correspondence between compact Riemann surfaces and function fields in one variable over $\boldsymbol{C}$ is well known and has been widely exploited, both in analysis and in algebraic geometry. A similar correspondence exists between function fields in one variable over $\boldsymbol{R}$ and compact "Klein surfaces," which are more general than Riemann surfaces in two respects: they need not be orientable and they may have boundary (see the precise definition below). But while this latter correspondence has apparently been in the folklore for some time, it seems to have been neither systematically expounded nor applied.

It was first noted by Felix Klein in 1882 [3] that nonorientable surfaces could carry an analytic structure. The analytic theory of Klein surfaces was treated in depth by Schiffer and Spencer in 1954 [4]. Our approach is quite different, since we endeavor to work within the category of Klein surfaces, rather than to lift problems to the category of Riemann surfaces by doubling. This approach allows us to prove certain results (such as Theorems 5 and 6 below) without resort to the technique of descent.

In this announcement we state the foundational definitions and theorems, and then give several applications, some of which appear to be new. A detailed monograph is in preparation.

1. Klein surfaces. Let $D$ be an open set in $C$ and let $f: D \rightarrow C$ be a $C^{2}$ function as a function of $R^{2}$ into $R^{2} . f$ is of course analytic if $d f / d \bar{z}$ $=0$ and antianalytic if $d f / d z=0$. We call $f$ dianalytic if on each component of $D$ it is analytic or antianalytic. If $D$ is an arbitrary subset of $\boldsymbol{C}$ and $f: D \rightarrow C$, we say that $f$ is dianalytic if there is a dianalytic extension of $f$ to some open neighborhood of $D$.

Let $X$ be a connected two-manifold with boundary $\partial X$ (which may be empty). A dianalytic coordinate covering (d.c.c.) of $X$ is a family $\mathfrak{U}=\left(U_{j}, z_{j}\right)_{j \in J}$, where $\left(U_{j}\right)_{j \in J}$ is an open cover of $X$, and $z_{j}$ is a homeomorphism of $U_{j}$ onto an open set in the closed upper half plane $C^{+}$, such that the transition functions $z_{j} z_{k}{ }^{-1} \mid z_{k}\left(U_{k}\right)$ are all dianalytic. A dianalytic structure on $X$ is a maximal d.c.c. $\mathfrak{X}$, and a Klein surface

1 This research was supported, in part, by NSF grant GP 7548 and NSF grant GP 7436. 
consists of the pair $(X, \mathfrak{X})$ and will often be denoted by $\mathfrak{X}$.

If $\mathfrak{X}$ and $\mathfrak{Z}$ are Klein surfaces, then a morphism is a continuous map $f: X \rightarrow Y$, with $f(\partial X) \subset \partial Y$ and with the following local behavior. Let $x \in X, y \in Y$, with $f(x)=y$. Then there exist $(U, z) \in \mathbb{X},(V, w) \in \mathscr{Y}$ with $f(U) \subset V$ such that $f \mid U=w^{-1} \circ \phi \circ h \circ z$, where $h$ is an analytic function on $z(U)$, and $\phi$ is the "folding map," $\phi(a+b i)=a+|b| i$. If $\mathfrak{X}$ and $\mathfrak{Y}$ are compact and $f: \mathfrak{X} \rightarrow \mathfrak{Y}$ is a nonconstant morphism, then of course $f$ is a surjection. We let $\mathscr{K}$ denote the category of compact Klein surfaces and nonconstant morphisms.

Let $\mathfrak{X}$ be a Klein surface with $\mathfrak{X}=\left(U_{j}, z_{j}\right)_{j \in J}$. By a meromorphic function $\mathfrak{g}$ on $\mathfrak{X}$ we mean a family $\mathfrak{g}=\left(g_{j}\right)_{j \in J}$ where $g_{j}: U_{j} \rightarrow C \cup\{\infty\}$, which satisfies

(i) $g_{j} \circ z_{j}^{-1}$ is meromorphic,

(ii) if $x \in \partial X$, then $g_{j}(x) \in R \cup\{\infty\}$,

(iii) if $V$ is a component of $U_{j} \cap U_{k}$ such that $z_{j} \circ z_{k}{ }^{-1} \mid z_{k}(V)$ is analytic (resp. antianalytic) then $g_{j}\left|V=g_{k}\right| V$ (resp. $\left.g_{j}\left|V=\bar{g}_{k}\right| V\right)$.

Let $E(\mathfrak{X})$ denote the set of all meromorphic functions on $\mathfrak{X}$. Then with the obvious definitions $E(\mathfrak{X})$ is a field extension of $R$. If $f: \mathfrak{X} \rightarrow \mathfrak{Y}$ is a nonconstant morphism, then there is induced an $R$-monomorphism $E(\mathfrak{Y}) \rightarrow E(\mathfrak{X})$.

THEOREM 1. If $\mathfrak{X} \in \mathrm{Ob}(\mathfrak{K})$, then $E(\mathfrak{X})$ is an algebraic function field of one variable over $R$.

It is easily seen that $E(\mathfrak{X})$ contains an isomorphic copy of $C$ if and only if $X$ is orientable and $\partial X=\varnothing$. A natural task is to seek algebraic criteria for orientability. A result of Ahlfors [1] yields

TheOREM 2. Let $\mathfrak{X} \in \mathrm{Ob}(\mathfrak{K})$ with $\partial X \neq \varnothing$. Then $X$ is orientable if and only if there is $a \mathfrak{f} \in E(\mathfrak{X})$ such that $\mathfrak{f}(x) \in R \cup\{\infty\}$ implies $x \in \partial X$.

2. Real algebraic function fields. Let $Q$ be the category of algebraic function fields in one variable over $\boldsymbol{R}$ (finitely generated extension fields of $\boldsymbol{R}$ of transcendence degree one) and $\boldsymbol{R}$-algebra monomorphisms. If $E \in \mathrm{Ob}(R)$, let $X \equiv \mathrm{Riem}_{R} E$ be the set of all valuation rings $\mathfrak{D}$ of $E$ which contain $R$. If $f \in E$, there is induced a map $\hat{f}$ from $X$ to the compactified upper half plane $C^{+} \cup\{\infty\}$, where $\hat{f}(\mathfrak{D})$ is the image of $f$ in the residue field of $\mathfrak{D}$, followed by the folding map.

If $X$ is given the weak topology making all $f$ continuous, it becomes a compact two-manifold and carries a canonical dianalytic structure $\mathfrak{X}$. Further $E$ and $E(\mathfrak{X})$ are canonically isomorphic. In sum, we have

THEOREM 3. $E$ is a contravariant functor from $\Re$ to $R$, and $\mathrm{Riem}_{R}$ is a contravariant functor from $R$ to $\mathcal{K}$. They are, up to natural equivalence, inverses of one another. 
EXAmple 0. Let $E$ have (algebraic) genus 0 . Then $E$ is isomorphic to $C(z), R(z)$, or $R(x, y)$ with $x^{2}+y^{2}=-1$. Hence $\operatorname{Riem}_{R} E$ is accordingly either the Riemann sphere, the compactification of the upper half plane (i.e., a closed disc), or the real projective plane (N.b., this proves very easily that all real projective Klein planes are dianalytically equivalent.)

ExAmple 1. Let $E$ have genus one. Then $\operatorname{Riem}_{R} E$ is topologically a torus, a Klein bottle, an annulus, or a Möbius strip. Examples of fields giving each of these are $C(x, y)$ with $y^{2}=x^{3}-x ; R(x, y)$ with $y^{2}=-\left(x^{2}+1\right)\left(x^{2}+2\right) ; R(x, y)$ with $y^{2}=x^{3}-x ;$ and $R(x, y)$ with $y^{2}=x^{3}+x$, respectively.

We remark that the various (inequivalent) dianalytic structures which can be put on the annulus, Klein bottle, or Möbius strip lie in each case in a one (real) parameter family.

3. Coverings and extensions. If $\mathfrak{X} \in \mathrm{Ob}(\mathfrak{K})$, then Theorem 3 yields a correspondence between morphisms $\mathfrak{Z} \rightarrow \mathfrak{X}$ in $\mathscr{K}$ and finite extensions of $E(\mathfrak{X})$. Given $\mathfrak{X}$, we may obtain every such $\mathfrak{Z}$, as a topological space, as follows. Let $\rho: \tilde{X} \rightarrow X$ be a ramified covering of $X$ of finite degree, with $\tilde{X}$ compact (and perhaps disconnected). We pass from $\tilde{X}$ to $Y$ by making a sequence of "permissible" identifications on $\partial \tilde{X}$. Namely, let $I$ be a closed arc in $\partial X$, and let $I_{1}, I_{2}$ be closed arcs in $\partial \tilde{X}$ such that $\rho$ maps $I_{j}$ homeomorphically onto $I$, and $I_{1}$ and $I_{2}$ are either disjoint or intersect at one or both endpoints. Then $I_{1}$ and $I_{2}$ may be identified in the obvious manner. If $\rho^{\prime}: X^{\prime} \rightarrow X$ is obtained in this manner, we call it a folded cover of $X$.

Theorem 4. If $\mathfrak{Y} \rightarrow X$ is a morphism in $\mathfrak{K}$, then it is (topologically) a folded cover of $X$. If $X^{\prime} \rightarrow X$ is a folded cover of $X$, then there is a unique Klein surface structure on $X$ making this a morphism in $\Re$.

Corollary. Let $X$ be a compact two-manifold with $r$ boundary components. Then $X$ can carry a dianalytic structure. Further, $X$ can carry a dianalytic structure $\mathfrak{X}$ with $E(\mathfrak{X})$ hyperelliptic [2], except in the case $r \geqq 3, X$ orientable, and $\chi(X)<2-r(\chi(X)$ denotes the Euler characteristic of $X)$.

The topological information in Theorem 4 yields, via Theorem 3, algebraic results. We first state a strengthened form of a theorem of Witt [5].

Theorem 5. Let $\mathfrak{X} \in \mathrm{Ob}(\mathfrak{K})$ and let $I_{1}, \cdots, I_{n}$ be disjoint closed intervals or complete components of $\partial X$. Then there is an $\mathrm{f} \in E(\mathfrak{X})$ such that $\mathrm{f}(x) \geqq 0$ for $x \in \cup_{j=1}^{n} I_{j}$, and $\mathrm{f}(x) \leqq 0$ for $x \in \partial X \backslash \cup_{j=1}^{n} I_{j}$. Further $f$ 
may be chosen so that it has zeros and poles of odd order only at the endpoints of the $I_{j}$.

Let $\mathfrak{X} \in \mathrm{Ob}(\mathcal{K})$ have $r$ boundary components, $r \geqq 1$, and let $\chi(X)$ denote its Euler characteristic. Then we have

THEOREM 6. There are $2^{a}-1$ unramified quadratic extensions of $E(\mathfrak{X})$, where $a=r+[1-\chi(X)]$. (Among these, of course, is $E(\mathfrak{X})(i)$ which corresponds to the classical double of $\mathfrak{X}$.)

Finally we note that the following theorem of Harnack is an easy consequence of our considerations:

Theorem 7. If $C$ is a real algebraic curve of genus $g$, then the real locus of $C$ has at most $g+1$ components.

\section{REFERENCES}

1. L. Ahlfors, Open Riemann surfaces and extremal problems on compact subregions, Comment. Math. Helv. 24 (1950), 100-134.

2. C. Chevalley, Introduction to the theory of algebraic functions of one variable, Math. Surveys, no. 6, Amer. Math. Soc., Providence, R.I., 1951; Russian transl., Fizmatgiz, Moscow, 1959.

3. F. Klein, Über Riemanns Theorie der algebraichen Funktionen und ihrer Integrale, Teubner, Leipzig, 1882.

4. M. Schiffer and D. Spencer, Functionals of finite Riemann surfaces, Princeton Univ. Press, Princeton, N. J., 1954.

5. E. Witt, Zerlegung reelor algebraischer Funktionen in Quadrate Schiefkörper uiber reellen Funktionen köeper, Crelles J. 171 (1934), 4-11.

UNIVERSITY OF ROCHESTER, ROCHESTER, NEW YORK 14627 